\title{
SIMPLIFIED PROCEDURE FOR THE NONLINEAR SEISMIC ANALYSIS OF BRIDGES
}

\author{
F. Montans and E. Alarcon \\ Department of Structural Mechanics and Industrial Constructions, Technical \\ University of Madrid, Madrid, Spain
}

\begin{abstract}
The severe accidents suffered by bridges during recent earthquake show that more careful analysis are needed to guarantee their behaviour. In particular simplified non-linear analysis could be useful to bridge the gap between theoretical research and practical applications. This paper presents one of those simplified methods that can be applied for first designs or to retrofitting of groups of bridges.
\end{abstract}

\section{Introduction and Objetives}

In Dynamics of Structures the selection of the computacional Method impinges directly on the accuracy and cost of a Dynamic Analysis. This is specially true in Seismic Engineering where it is usually accepted that a certain amount of damage can happen; for instance damage is accepted in bridges if after the shock the structure can sustain the emergency traffic. That implies the use of non-linear analysis.

The computational methods generally in use are:

- Equivalent static procedures

- Modal superposition or spectral analysis

- Step by step integration in the time domain

The advantage of the first group of procedures is their simplicity and this is why they were adopted in Seismic Codes. The main disadvantage is related to the approximate character of their results and as a consequence, they are randomly distributed around true values.

The developments in computer hardware and software, as well as those related to the rationalization of spectra have contributed to the increase of modal computations that, being generally based on a linear approach combined with rules related to mode truncation and combination as well as to global non-linear behaviour through ductility factors can only be used in structures relatively regular where non-linear effects are well distributed. They can not describe the evolution of the structure along with the seismic action what in some occasions can be crucial, for instance when there are zones with different ductilities or, more often, when it is necessary to make estimations of the ductility demand. All those difficulties are overpassed thanks to the step by step methods which due to the effort needed are only used for special structures.

For bridges, in addition to the occasion in which an individual bridge has to be carefully studied, there exists the possible need of a repetitive study on a set of bridges to analize the need of retrofitting and to identify potential areas in which a relatively fine study is needed. In those situations one is confronted with the need of reducing the duration and cost of the analysis what proscribes the use of a very complicated model.

This is why this paper is dedicated to analyse the possible advantages of a simplified method that could combine the advantages of the step by step methods with fast and simple computations easy to follow by practising engineers and uselful for parametric studies in order to quantify the effects of the different retrofitting measures.

The motivating idea was published as an Annex to one of the drafts of EUROCODE 8 Part II ref.[1] although the origins can be found in the famous Bigg's book ref.[2]. The computations shown here follow the philosophy of that approach although some modifications or alternatives are introduced in order to improve their performances.

In the end of the paper several examples on regular and irregular bridges are used to show the possibilities and limitations of the method.

\section{The Dynamic Plastic Hinge Method for Framed Structures}

\subsection{General scheme}

It is intended to obtain the structural response using a single degree of freedom system related to the main vibration model or to a static deformation collecting the main contribution of the eigenmodes in the direction of the seismic action.

The shape function is evolutionary in the sense that plastic hinges can be formed during the earthquake and therefore a new shape has to be taken to reproduce the displacements.

The method is based on the following points:

- Use of a evolutionary vibration period to represent the dynamic response of the structure

- Assuming that the hinges can be produce only in the end of the member sections and that the behaviour is perfectly elastoplastic

- Modifying the structural model as it is being degraded, changing the shape function, the stiffness and obtaining the response as a combination of a sequence of Equivalent Substituting System

The moments in which the structure changes its configuration are identified through its acceleration and can be collected in a poligonal line that is called "Modal Lodad-Deflection Line (MLDL)". 


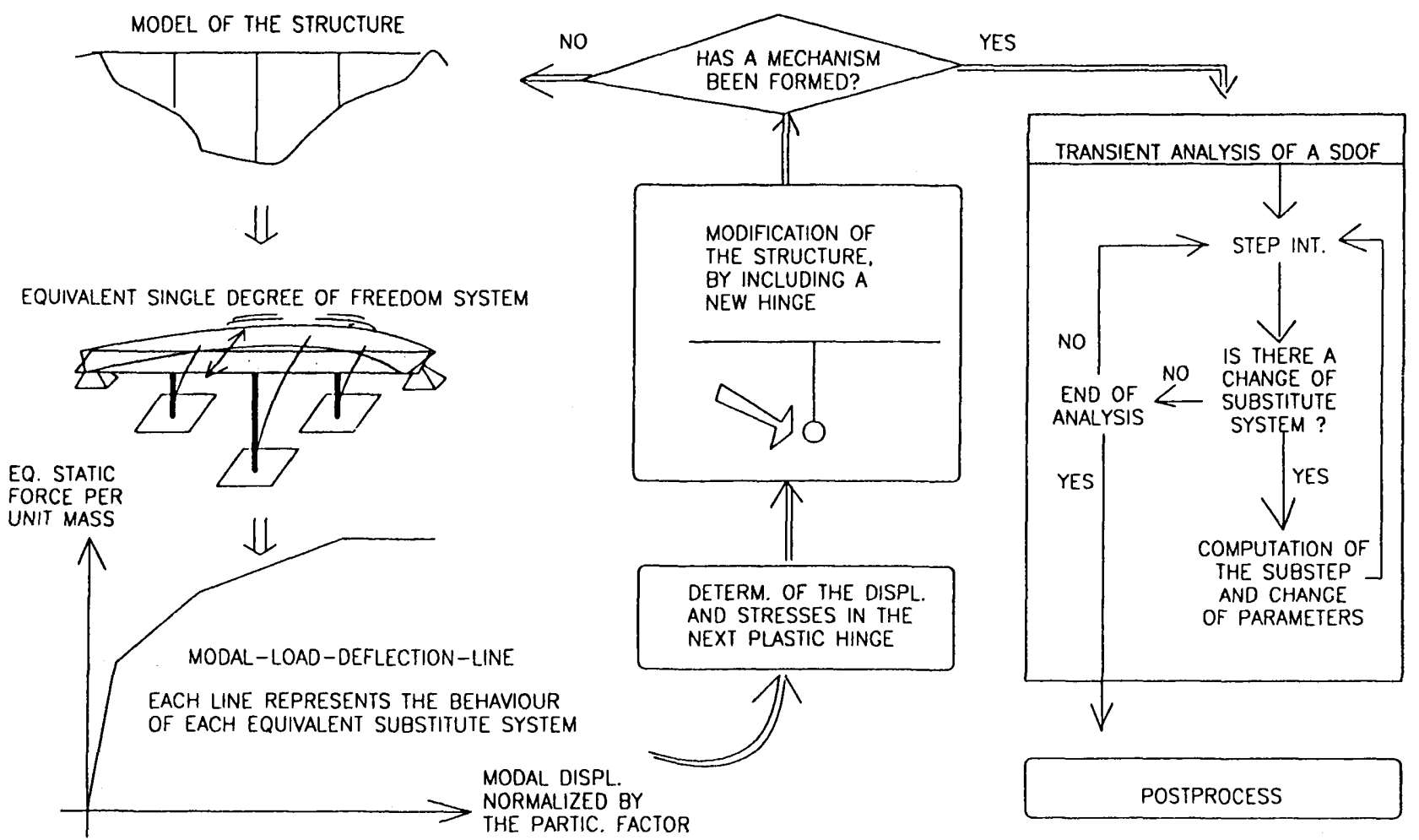

Figure 1. Analysis procedure

As can be deduced from the previous lines the analysis is organized into three blocks (figure 1)

a) Computation of the MLDL identifying the substitute systems through which the structure can pass until last configuration

b) Transient step by step analysis of the equivalent evolutionary one degree-of-freedom system

c) Postprocessing. Computation of stresses and displacements at different points of the structure

To obtain the equivalent system (a) it is necessary to use three types of computations:

a.1.) Modal shape and frequency vibration. This has been done using two alternatives: by classical eigenvalue analysis choosing the mode with higher participation factor and by Rayleigh method using the static deformation of the structure subjected to a force proportional to the mass distribution and acting in the direction of the earthquake as a shape function

a.2.) Computation of the static forces produced in every degree of freedom by a unit acceleration so that a multiple of eigenvalue is obtained

a.3.) Computation of the forces and displacements at different points of the structures. With the knowledge of the plastic moment, it is easy to determinate the localization of the next plastic hinge

Once the new hinge is produced the system will change its behaviour increasing the displacements but mantaining the level of efforts at the yielded section. The new configuration incorporating the new hinge is the Substitute System with which the analysis will be continued.
Going back to a.1) the behaviour of the new system is computed until all pieces of the MLDL curve have been obtained. After that it is only necessary to use an step by step integration method to obtain the response of the equivalent single-degree-of-freedom system. In that respect it is interesting to localize the stiffness changes in the exact form by using a consistent event localization technique (CELT), as described for instance in ref.[5] to detect either the unloading or the formation of a new plastic hinge (overloading).

Finally the post processing (c) does not present any special difficulty because the response in terms of a generalized one is already known as it is equivalent substitute system, that allows the use of typical techniques of matrix analysis for the computation of member forces and displacementes, as well as the rotation of every plastic hinge.

In particular the knowledge of the plastic hinge rotations allows the knowledge of local ductility demands and the computation of several damage measures.

\subsection{Structural Modelling}

To model the structure the following statements can be considered:

- The stiffness matrix for a static analysis is "exact" and independent of the discretization

- The lumped mass matrix depends on the discretization and improves with fines meshes

- The computational time and the memory required increase slightly with the mesh refinement because the transient analysis is independent of the number of elements, although the establishment of the 
equivalent substitute system is mesh-dependent. It is worthwhile to point out that the use of Rayleigh method in place of a modal analysis is more convenient in terms of computational time and memory requirements.

\subsection{Computation of the Equivalent Substitute Systems}

To establish the line governing the behaviour of the equivalent 1 d.o.f. it is convenient to start with the equilibrium equation of the $n$ d.o.f. structure

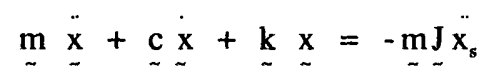

where $\mathrm{m}, \mathrm{c}$ and $\mathrm{k}$ are repectively the mass, damping and stiffness matrices. The $\underset{\sim}{\mathrm{x}}, \underset{\sim}{\dot{\mathrm{x}}}$ and $\underset{\sim}{\ddot{\mathrm{x}}}$ are respectively displacements, velocity and acceleration vectors of structural d.o.f., $\mathrm{J}$ is the influence vector resulting from the projection of the d.o.f. on the earthquake direction and $\ddot{x}_{s}(t)$ the earthquake accelerogram. As it is well know the modal projection

$$
\underline{x}=\underline{\Psi} \xi
$$

(where $\psi$ is the matrix which columns are the eigenvectors and $\xi$ are the modal coordinates) produces in the case of classical damping, an uncoupled system. If a certain mode $\underset{\sim}{\phi}$ is predominant, it is posible to write

$$
\underline{\mathbf{x}} \approx \boldsymbol{\xi} \phi
$$

and only the following relationship

$$
\ddot{\xi}+2 \zeta \omega \dot{\xi}+\omega^{2} \xi=\Gamma \ddot{x}_{s}
$$

(where $\zeta$ is the damping ratio, $\omega$ the eigenfrequency:

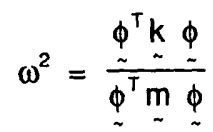

and $\Gamma$ the participation factor)

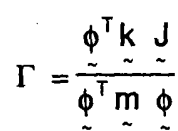

is required to approximately represent the structural response.

In Rayleigh method, the starting point is relationship (3) where $\phi$ is a reasonable estimate of the response that in our case will be obtained by loading the structure proportionally to the mass distribution in the direction of the earthquake.

To avoid the influence of different configuration it is advisable to write equation (4) as

$$
\left(\frac{\dot{\xi}}{\Gamma}\right)+2 \zeta \omega\left(\frac{\dot{\xi}}{\Gamma}\right)+\omega^{2}\left(\frac{\xi}{\Gamma}\right)=-\ddot{x}_{s}
$$

where $\left(\frac{\xi}{\Gamma}\right)$ represents displacements continuos from a substitute system to other one. In addition, the grouping

$$
\left[\left(\frac{\bar{\xi}}{\Gamma}\right)+\ddot{x_{s}}\right]+2 \zeta \omega\left(\frac{\dot{\xi}}{\Gamma}\right)=-\omega^{2}\left(\frac{\xi}{\Gamma}\right)
$$

allows the interpretation of the right hand side as a pseudoaceleration as plotted in figure 2 . The abcissa $\left(\frac{\xi}{\Gamma}\right)$ is the generalized coordinate and the vertical axis represents the pseudoaceleration or equivalent static force per unit mass. Every straight line will be valid until the response includes a new plastic hinge.

From here on the member forces can be computed using the equivalent static forces in static analysis and fixing an upper-threshold " a posteriori" by a convenient scaling.

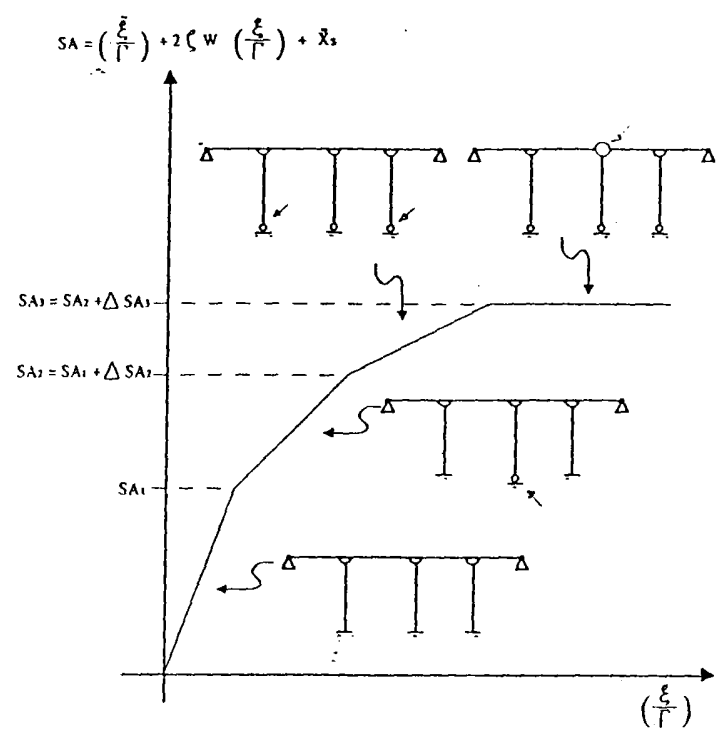

Figure 2. Example of the Modal-Load-Deflection-Line

\subsubsection{Change of the structural model}

Once the plastic moment of a section has been reached the structural response changes due to the formation of a hinge for the incremental loads. In the hereby presented approach the behaviour of the sections is assumed to be perfectly elastoplastic. Then, it is necessary to modify the stiffness matrix of the member in which the hinge is formed using techniques well known in matrix structural analysis.

The MLDL is obtained for every situation as shown in figure 2 . If is assumed that the curve is symmetric with respect to the origin, what implies, among other things, symmetric reinforcement of concrete sections.

Despite of other ones, it is possible to make the following considerations:

- The straight line representing the behaviour of the substituting system (n) starts at the point of system (n-1), and system (1) starts from the origin. Superposition is applied in an incremental fashion, so that is $\mathrm{SA}_{1} ; \mathrm{SA}_{2} \ldots$ $\mathrm{SA}_{\mathrm{n}}$ are the pseudoaccelerations for which the plastic hinges $1,2, \ldots \mathrm{n}$ appear the member forces and displacements for a load

$$
\mathrm{SA}_{\mathrm{i}=1}<\mathrm{SA}^{*}<\mathrm{SA}_{\mathrm{i}}
$$

wil be 


$$
\begin{aligned}
& \text { Axial force } N^{*}=n^{i}\left(S A^{*}-S A_{i-1}\right)+N^{i-1} \\
& \text { Shear force } V^{*}=v^{i}\left(S A^{*}-S A_{i-1}\right)+V^{i-1} \\
& \text { Bending Moment } M^{*}=m^{i}\left(S A^{*}-S A_{i-1}\right)+M^{i-1} \\
& \text { Displacement } D^{*}=d^{i}\left(S A^{*}-S A_{i-1}\right)+D^{i-1}
\end{aligned}
$$

where $\mathrm{N}^{\mathrm{i}-1}, \mathrm{~V}^{\mathrm{i}-1}, \mathrm{M}^{\mathrm{i}-1}$ and $\mathrm{D}^{\mathrm{i}-1}$ are the forces and displacements when the substituting system (i-1) was left and $\mathrm{n}^{\mathrm{i}}, \mathrm{v}^{\mathrm{i}}, \mathrm{m}^{\mathrm{i}}$ and $\mathrm{d}^{\mathrm{i}}$ the efforts and displacements for a unit acceleration in the substituting system (i)

- As the pseudoaccelerations at the starting point of every substituting system are

$$
\Delta S A=\Delta \ddot{X}_{s}+\left(\frac{\dot{\xi}}{\Gamma}\right)+2 \zeta \alpha\left(\frac{\dot{\xi}}{\Gamma}\right)
$$

it is seen that it depends on the value taken for $\zeta$. At one point of MLDL curve before the formation of the plastic hinge if it is possible to write

$$
S A^{(-)}=\Delta \ddot{X}_{S}+\Delta\left(\frac{\dot{\xi}}{\Gamma}\right)+2 \zeta^{\prime} \omega^{\prime} \Delta\left(\frac{\dot{\xi}}{\Gamma}\right)+S A_{i-1}
$$

where $z^{i}, w^{i}$ and $G^{i}$ are the damping ratio, natural frecuency and participation factor for system $i$; and $\Delta \bar{x}_{s}, D\left(\ddot{\xi} / G^{i}\right)$ and $D\left(\xi / G^{i}\right)$ are the increments of soil accelerations and velocities since the formation of the plastic hinge.

Then, it is seen that different damping ratios can be chosen for different configurations as an alternative to the usual assumption of a constant damping ratio throughout the whole time interval.

\subsubsection{Computation of the main eigenvalue and eigenvector}

As can be deduced from the last paraghaps, one key factor for the procedure is the selection of a shape function $f$ (eq. 3). Two ways have been chosen to do so:

- An eigenvalue analysis by the subspace iteration mode selecting the one with higher mobilized mass, what is repeated for every substituting system

- A Rayleigh method using the static deflections produced by a load proportional to the mass matrix acting along the earthquake direction.

It has been found that the last method is more convenient from a computational viewpoint and, as the parametric study has shown, the results are comparable and in occasions better than using the main mode.

The steps needed to determine the load-displacement curve (MLDL line) can be summarized in the following points:

- An eingenvalue analysis of the configuration (wich includes the existing plastic hinges) or a static analysis is conducted to determine the selected shape function $\phi$ and its associated value

$$
\omega^{2}=\frac{\underline{\phi}^{\top} k \underset{\sim}{\phi} \underline{\sim}}{\underline{\phi}^{\top} \underline{\dot{m}} \underline{\sim}}
$$

- The equivalent static forces per unit acceleration are computed using the shape vector $\phi$
- A static analysis under the above mentioned forces is conducted

- The response acceleration of the structure capable of producing the next plastic hinge is computed. The new points of the MLDL line will be

$$
\begin{aligned}
& \left(\frac{\xi}{\Gamma}\right)=\left(\frac{\xi}{\Gamma}\right)^{-1}+\frac{\Delta S A_{1}}{\omega^{2}} \\
& S A_{n}=S A_{t-1}+\Delta S A_{i}
\end{aligned}
$$

- Using the previous accelerations new member forces and displacements are computed

- The new substituting system is modeled

- If the new system is not a mechanism the process is repeated. In our case the deck is assumed to remain in the elastic range so the procedure is finished when all the possible plastic hinges have developed in the piers.

\subsection{Transient analysis of the equivalent s.d.o.f. system}

As soon as the equivalent system have been determined it is possible to obtain the MDLD curve governing of the s.d.o.f. subjected to the action of a specific accelerogram. One of the advantages of the method is the linear behaviour between events, so that a simple Newmark-b method can be used along with the Consisten Event Location Technique (CELT) described in ref. [5].

\subsection{Postprocessing. Computation of displacements, rotations and member forces}

The transient analysis provides the response in nodal coordinates. i.e.: the displacements histories $x_{j} / G_{i}$ the pseudoaccelerations $S A_{i}$ and the equivalent system $\mathrm{j}$ valid at each instant are known. As shown in figure 3 it is and easy matter to obtain the time histories of displacements, member forces, etc: Once the configuration $i(t)$ is known, following the previous notation it is possible to write

$$
\begin{aligned}
& \underset{\sim}{X}(t)=\sum_{i=0}^{t} \Phi_{i(t)} \Delta\left(\frac{\xi}{\Gamma_{1}}\right)^{(t)} \Gamma_{i(t)} \\
& \underset{\sim}{N}(t)=\sum_{t=0}^{t} \underset{\sim}{n} \Delta S A(t) \\
& \underset{\sim}{M}(t)=\sum_{t=0}^{t}{\underset{\sim}{i(t)}}_{(S A} \Delta(t)
\end{aligned}
$$

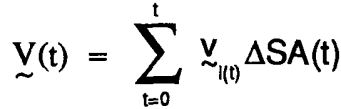

\subsection{Implementation of the method in a computer program}

To develop a study in order to validate the method, a program called AROSA written in Fortran VMS has been developed. The global process is shown in Figure 4 and has four modules: Input, Computation of the MDLD line, transient analysis and postprocessing, all of them following the scheme developed in the previous points. 


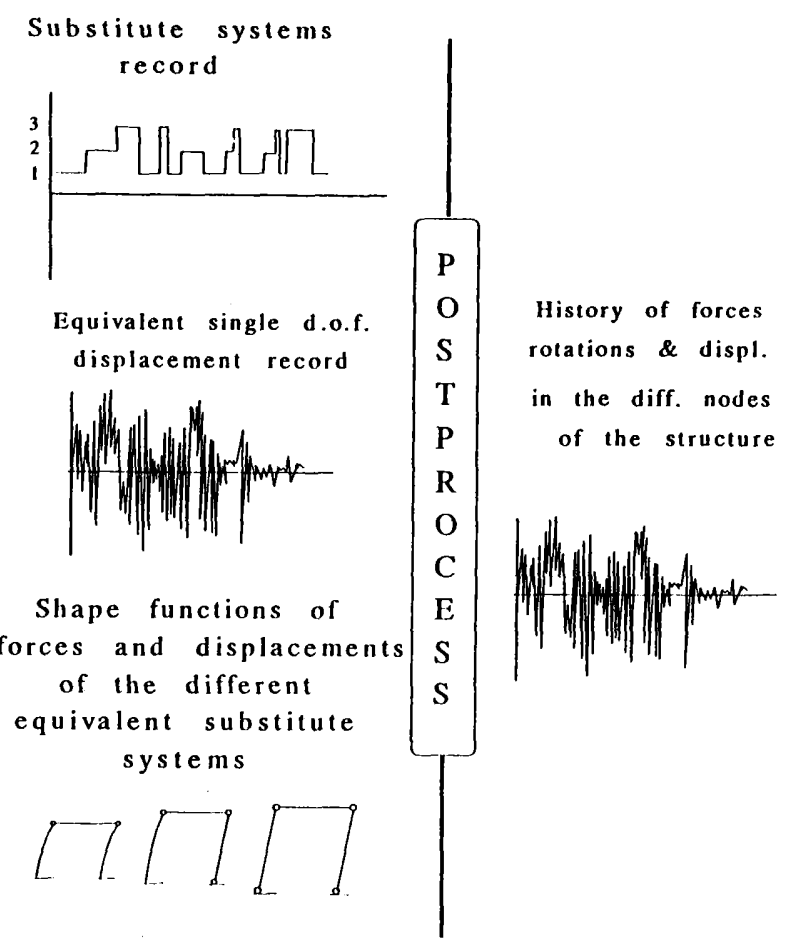

Figure 3. Postprocessing

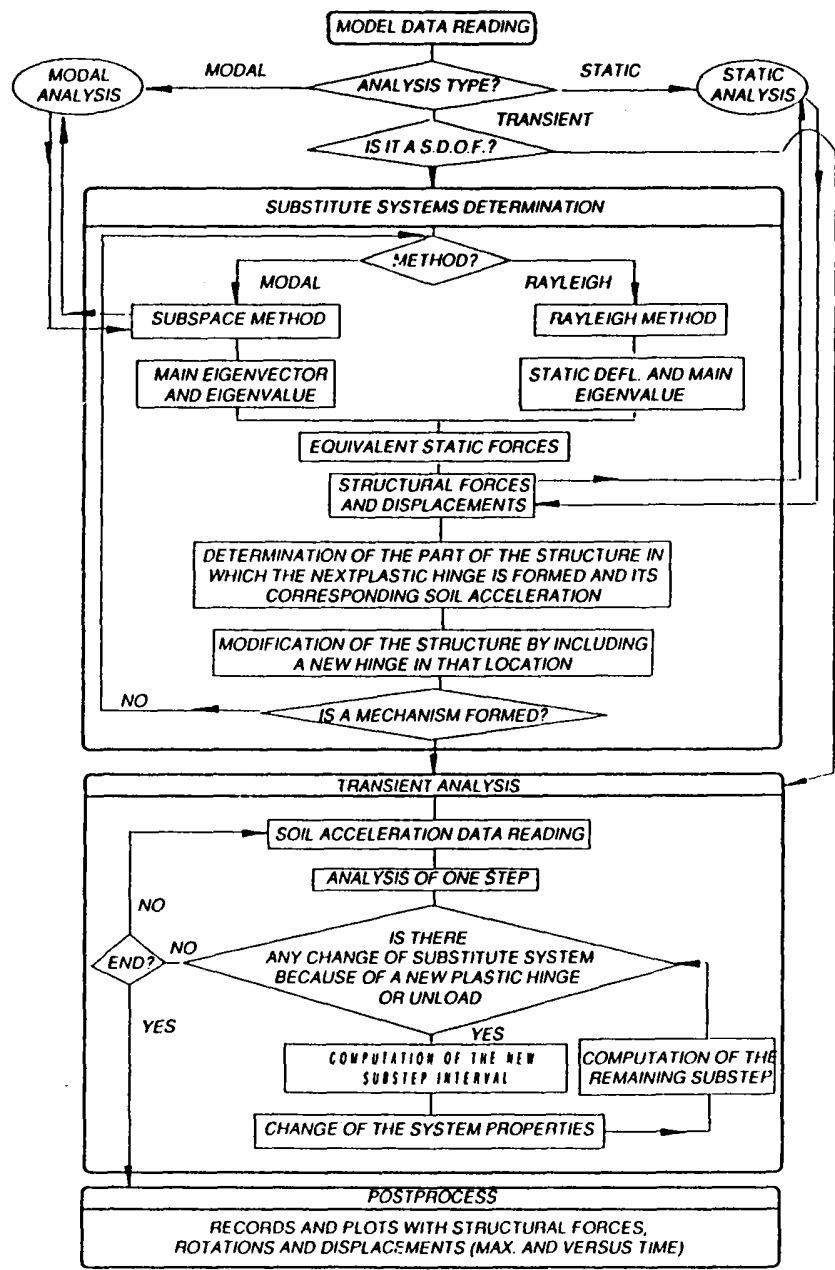

Figure 4. Scheme of the implementation of the DynamicPlastic-Hinge-Method

\section{Examples}

To analize the validity of the method several earthquakes were simulated and the two alternatives above mentioned were checked one against each other and also against the results obtained using a classical Finite Element approach using ANSYS commercial program.

Several checks were also done using DRAIN 2D code. The bridge models were those proposed by the Prenormative Research on Eurocode 8 (PREC-8) Committee to calibrate part 2 of that EC. The structures are continuous deck bridges with three piers with different heigh and reinforcement but with identical cross section. Figure 5 shows the examples studied. The so-called 232 bridge has a "regular" behaviour because its geometry favours a response very similar to that of the one dech alone (ie.: the first vibration mode is expected to be predominant). On the other hand, bridges $213 \mathrm{x}$ have a geometry provoking a very "irregular" behaviour. To analyse the effect of the amount of reinforcement in the bridge response, different degrees of reinforcement have been used in the models called $213 \mathrm{~A}, 213 \mathrm{~B}$, and $213 \mathrm{C}$. Bridge $213 \mathrm{~A}$ follows the usual criteria $(0.50-0.92-0.50 \%)$; Bridge $213 \mathrm{~B}$ is used to analize the increase of reinforcement in the central pier $(0.50-$ $1.69-0.50 \%$ ) while maintaining the lateral ones and bridge $213 \mathrm{C}$ is used to see the effect of lateral piers very reinforced and the central one with lower reinforcement (1.15-0.50-1.15\%).

In the developed study, two groups of earthquakes were used; four historical ones

$$
\begin{array}{ll}
\text { - TER 1: } & \text { El Centro } 1940 \\
\text { - TER 4: } & \text { San Fernando } 1971 \\
\text { - TER 5: } & \text { Imperial Valley } 1979 \text { (James Rd.) } \\
\text { - TER 6: } & \text { Imperial Valley } 1979 \text { (Bonds Corner) }
\end{array}
$$

and seven artificial ones (ACC 1 to ACC 7) that were generated using the program SIMQKE and were compatible with the EC-8spectra used for the modal analysis.

The natural frecuencies and the mobilized masses of the first three modes can be seen in Table I.

Tabla 1. Eigenvalues and mobilized masses of the analyzed bridges.

\begin{tabular}{|c|c|c|c|c|c|c|}
\hline \multirow{2}{*}{ Bridge } & \multicolumn{2}{|c|}{ MODE 1 } & \multicolumn{2}{c|}{ MODE 2 } & \multicolumn{2}{c|}{ MODE 3 } \\
\cline { 2 - 7 } & $\begin{array}{c}\text { Freq. } \\
(\mathrm{Hz})\end{array}$ & $\begin{array}{c}\text { Mobiliz } \\
\text { mass. }\end{array}$ & $\begin{array}{c}\text { Freq. } \\
(\mathrm{Hz})\end{array}$ & $\begin{array}{c}\text { Mobiliz } \\
\text { mass. }\end{array}$ & $\begin{array}{c}\text { Freq. } \\
(\mathrm{Hz})\end{array}$ & $\begin{array}{c}\text { Mobilizm } \\
\text { ass. }\end{array}$ \\
\hline 232 & 1.164 & $87.8 \%$ & 3.953 & $10.8 \%$ & 10.38 & $1.4 \%$ \\
\hline $213 \mathrm{~A}$ & 1.885 & $12 \%$ & 2.263 & $84.9 \%$ & 10.79 & $2.8 \%$ \\
\hline 213 B & 1.897 & $6.5 \%$ & 2.394 & $89.9 \%$ & 10.94 & $3.3 \%$ \\
\hline 213 C & 1.919 & $30.0 \%$ & 2.276 & $67.6 \%$ & 10.72 & $2.4 \%$ \\
\hline
\end{tabular}

It is interesting to see that the predominant part of the mobilized mass corresponds generally to one mode wich shape can be approximated by a static loading although only for that symmetric bridge the first mode is predominant.

To compare the answers obtained by different methods the following magnitudes have been selected:

\section{- maximum displacement and rotations}



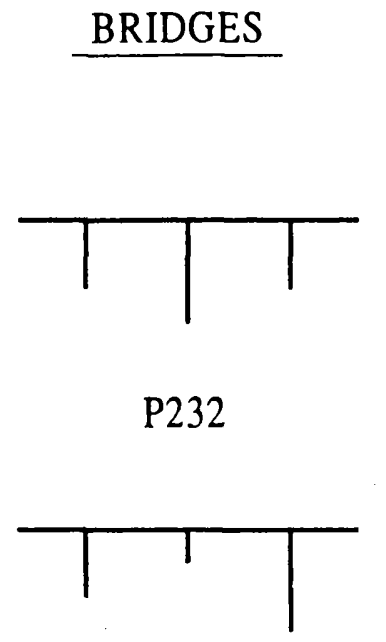

A213

B213

$\mathrm{C} 213$

Damping $=0 \%$

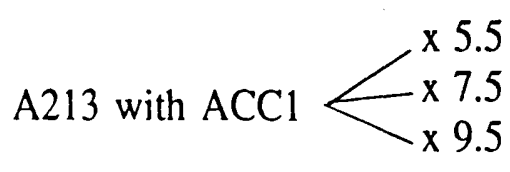

ACCEL. RECORDS
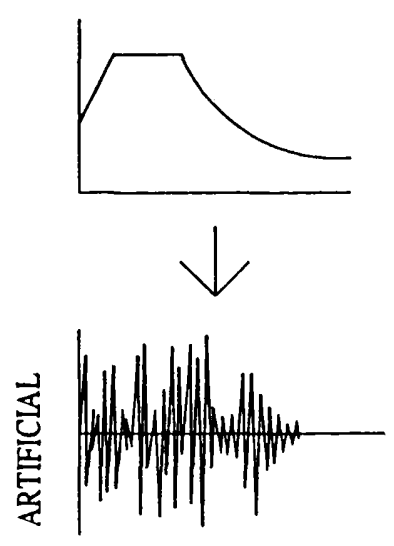

$\mathrm{ACC} 1 \ldots \mathrm{ACC} 7$

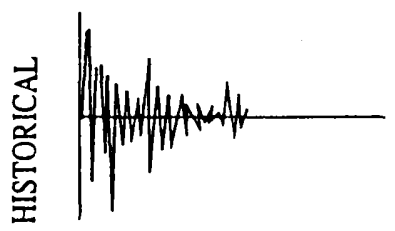

TER1: El Centro 1940

TER4: San Fernando 1971

TER5: Imperial Valley 1979

TER6: Imperial Valley 1979 (B.C.)

DISPLACEMENTS AT
EACH PIER TOP

ROTATIONS OF THE PLAS-

TIC HINGES

DUCTILITY DEMANDS

ENERGY DISIPATED BY

O THE PLASTIC HINGES

MAXIMUM POWER DIS.

BY THE PL. HINGES

Figure 5. Analyzed ridges
- ductility demands at plastified sections

- dissipated energy at plastic hinges

- member forces

In the following figures the comparison between the results obtaines using AROSA program (Dinamic plastic hinge method) and FEM ANSYS V5.0 is shown.

Figure 6.a shows the comparison between FEM and Rayleigh approach for the rotations at the plastic hinge in the central pier bridge 232 . It is seen that AROSA produces a very good aproacch of maxima, minima and general time-histories of the measures in all piers.

In other bridges the results are not so clear. Figure 6.b. shows the comparisonfor bridge $312 \mathrm{~A}$ and earthquake ACC 1 . Although larger differences than for the symmetric hinges are detected, it is possible to establish the following facts related to the simplified method

- Peak values are registred at exact time instants and the values fit in an acceptable way to the correct ones.

- In the intervals in which the differences are larger, the higher modes are excited by the accelerogram so the proportional contribution of the main mode-shape is different. Nevertheless the amplification of those modes seems to contribute only slightly to the maximum responses.

- It can be said that when the plastification is important the differences are reduced.
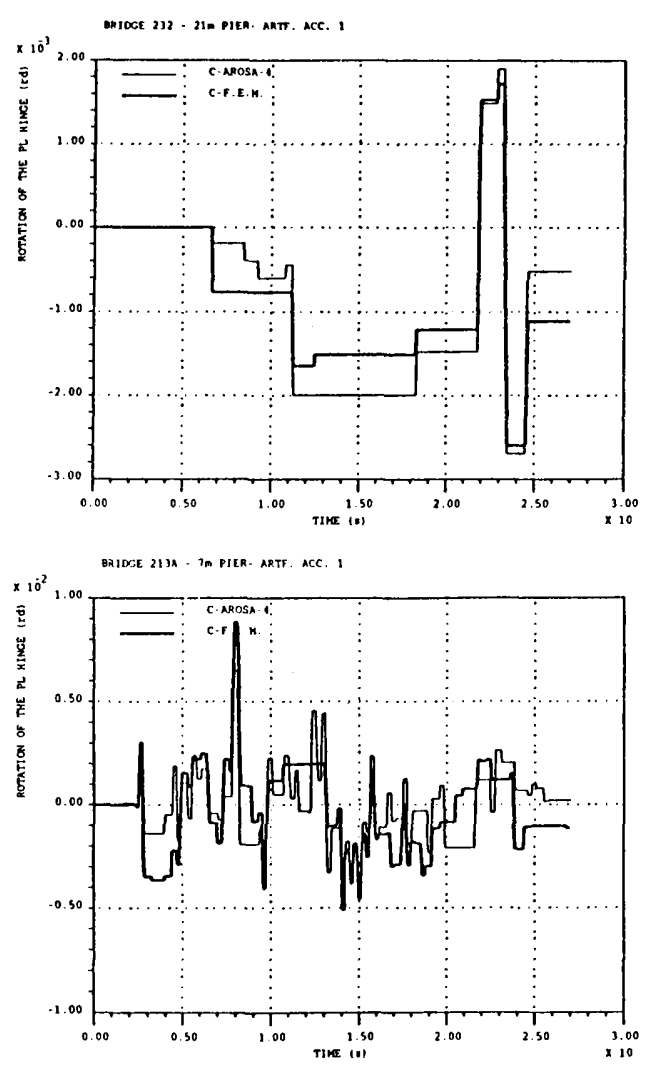

Figure 6. Comparison FEM versus AROSA. Rotations at the base of bridge 232 central pier (above) and 213A (below) due to the $\mathrm{ACCl}$ artificial accelerogram record. 
COMPARISON FEM.vS.AROSA

BRIDGE 213A-Maximum displacement

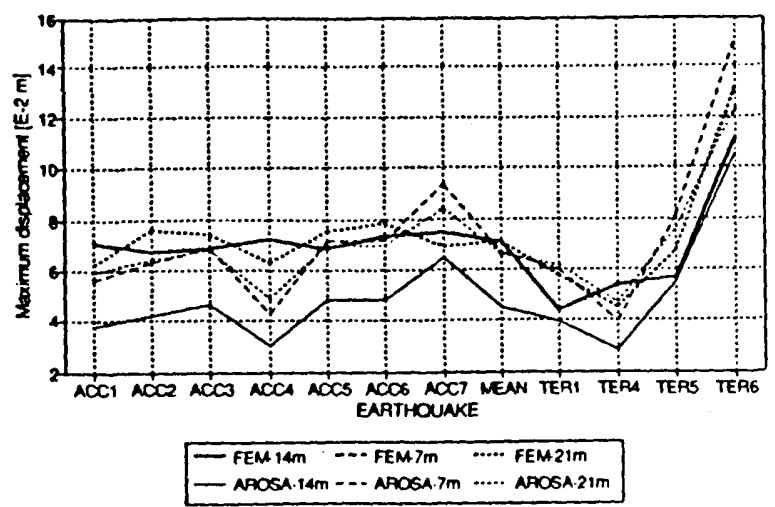

COMPARISON FEM.vS.AROSA

BRIDGE B213 - Maximum displacement

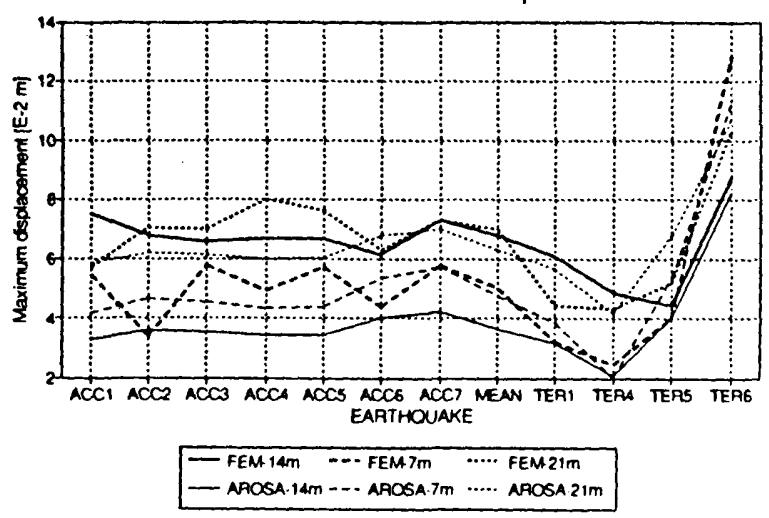

COMPARISON FEM.vS.AROSA

BRIDGE 232 - Maximum displacement

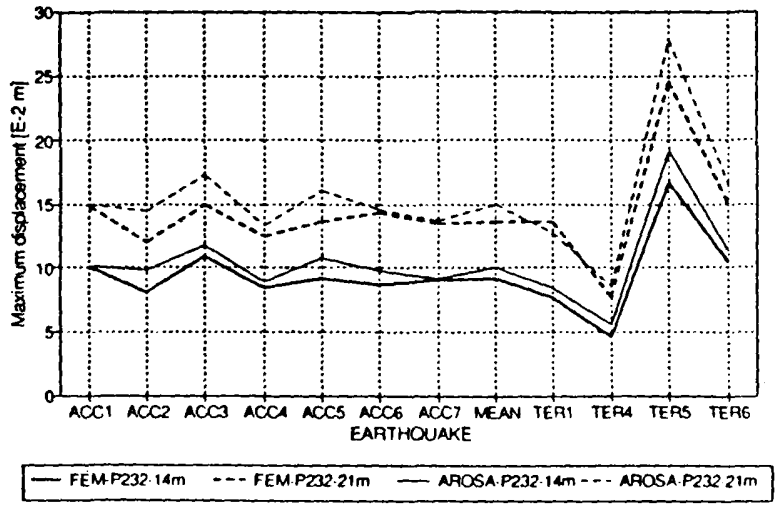

Figure 7. Comparison FEM vs. AROSA (Finite Element Method-Dynamic Plastic Hinge Method). Maximun displacements due to the different accelerograms.

Figure 7 shows the comparison of the maximun displacements at the top of the piers. In abcisas we represent the earthquake and in vertical axis the maximun values obtained. On those plots (plotted with continuous line for convinience) it is possible to see that the differences among computational methods are less than due to the use of different accelerograms.
It is also interesting to see that bridge $213 \mathrm{C}$, that in principle could be problematic because the used mode mobilizes less mass than the others, presents a very good accuracy in the rotation of the plastic hinge of the central pier, and with conservative values. More examples can be seen in ref. [7]

\section{Conclusions}

From the observed resultys and, at least, for the analysed tipology it is possible to conclude that:

- The D.P.H.M. produces displacements, rotations and energy histories very close to that of more accurate methods specially if one considers the variability induced by the excitations.

- In relation to the sections with greater ductility demands or dissipated energy the DPHM is a conservative method.

- The DPHM produces realistic degradation mechanisms that are very useful for a analysis in the case of seismic retrofitting. In those applications the method can be recomended as an analytical tool to discriminate which bridges need a more in-depth study and in which it is possible to accept the indications on the damaged areas.

\section{Acknowledgements}

This paper has been possible thanks to grant $n^{\circ} 93-0201$ of the Spanish DGICYT Foundations for Science and Technology

\section{References}

[1]

---: "Eurocode 8. Structures in Seismic Regions-Design Part2-Bridges". Commission of the European Communities. Borrador de 1990.

---: BIGGS. J. "Introduction to Structural Dynamics" McGraw Hill 1964

[3] Alarcón, E.; Álvarez, R.;Gómez Ma S.: "Matrix Analysis of Structures " (in spanish). Ed. Reverté. 1986

[4] Bathe, K.J. : "Finite Element Procedures in Engineering Analysis". Ed. Prentice-Hall. 1982

[5] Bernal, D. : "Locating Events in Step-by-Step Integration of Equations of Motion". Journal of Structural Engineering (ASCE) vol.117 n². Febrero 1991.

[6] Calvi,G.M.; Pavese,A.; Moncecchi,P.: "Progettazione in Zona Sísmica Di Ponti ad Impalcato Continuo". Universitá Degli Studi di Pavia. Febrero de 1994.

[7] Montans, F.'Ductility of Bridges under Seismic Loading. Application to seismic retroffiting" (in Spanish). Ph D thesis. Technical University of Madrid U.P.M., 1994. 